Somnologie $2016 \cdot 20: 241$

DOI 10.1007/s11818-016-0089-x

(c) Springer-Verlag Berlin Heidelberg 2016

CrossMark

\section{A. A. Schlarb ${ }^{1}$ B. Schneider ${ }^{2}$ M. Arzt ${ }^{3}$}

${ }^{1}$ Fakultät für Psychologie und Sportwissenschaft, Universität Bielefeld, Bielefeld, Deutschland ${ }^{2}$ Zentrum für Neuropädiatrie und Schlafmedizin, Kinderkrankenhaus St. Marien, Landshut, Deutschland ${ }^{3}$ Schlafmedizinisches Zentrum der Klinik und Poliklinik für Innere Medizin II, Pneumologie Universitätsklinikum Regensburg, Regensburg, Deutschland

\title{
Wenn Kinder nicht schlafen können
}

Kinder sind keine kleinen Erwachsenen - dies zeigen auch die Erkenntnisse aus der pädiatrischen Schlafforschung und Schlafmedizin.

Ein Beleg dafür sind ebenfalls die zahlreichen Beiträge, welche für das letzte Themenschwerpunktheft eingereicht wurden. Eine Auswahl zu treffen fiel schwer, und so sind wir dem Verlag und den Verantwortlichen sehr dankbar für die Möglichkeit, ein zweites pädiatrisches Schwerpunktheft folgen lassen zu können.

Viele Erkrankungen, die sich in der Kindheit manifestieren, haben einen Einfluss auf die Schlafqualität und damit Folgen für die Entwicklung der Kinder. Manchmal ist eine Schlafstörung aber auch das erste Symptom einer organischen oder psychischen Dysregulation. Diese wechselseitige Beeinflussung von Erkrankungen und Schlafverhalten sowie der familiäre Kontext spielen eine große Rolle in der Betrachtung pädiatrischer Schlafstörungen.

In den Arbeiten und Fallbeispielen werden diese Zusammenhänge, welche nicht immer gleich offensichtlich sind, herausgearbeitet und bieten einen weiteren Einblick in die vielfältigen Aufgabenstellungen der pädiatrischen Schlafmedizin.
Als Herausgeber dürfen wir uns bei den Autoren und Gutachtern für die gute und sehr engagierte Zusammenarbeit bedanken. Dieser Motivation ist es zu verdanken, dass wir ein zweites Schwerpunktheft präsentieren können. Ihnen als Leser dürfen wir eine informative und bereichernde Lektüre wünschen, die Ihren Blick für die nicht ganz kleinen Probleme unserer jungen Patienten schärfen möchte.

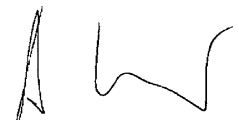

Angelika A. Schlarb

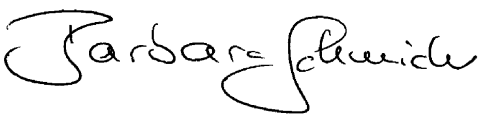

Barbara Schneider

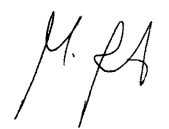

Michael Arzt
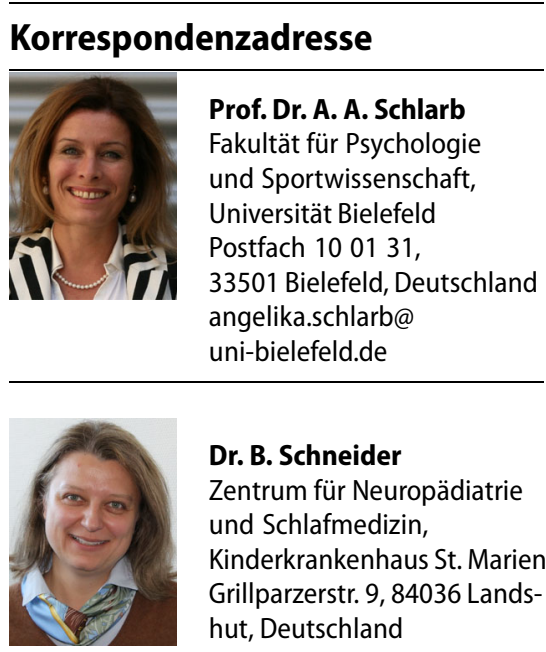

Dr. B. Schneider

Zentrum für Neuropädiatrie und Schlafmedizin, Kinderkrankenhaus St. Marien Grillparzerstr. 9, 84036 Landshut, Deutschland Barbara.Schneider@ st-marien-la.de

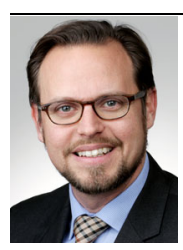

Prof. Dr. M. Arzt

Schlafmedizinisches Zentrum der Klinik und Poliklinik für Innere Medizin II, Pneumologie Universitätsklinikum Regensburg Franz-Josef-Strauß-Allee 11, 93053 Regensburg,

Deutschland michael.arzt@ klinik.uni-regensburg.de

Interessenkonflikt. A. A. Schlarb, B. Schneider und M. Arzt geben an, dass kein Interessenkonflikt besteht. 